\title{
Estimation of DOA for Noncircular Signals via Vandermonde Constrained Parallel Factor Analysis
}

\author{
Heyun Lin $\mathbb{D}^{1}{ }^{1}$ Chaowei Yuan, ${ }^{1}$ Jianhe Du, ${ }^{2}$ and Zhongwei Hu${ }^{1}$ \\ ${ }^{1}$ School of Information and Communication Engineering, Beijing University of Posts and Telecommunications, Beijing 100876, China \\ ${ }^{2}$ School of Information and Engineering, Communication University of China, Beijing 100024, China \\ Correspondence should be addressed to Heyun Lin; forlan@qq.com
}

Received 8 June 2017; Revised 21 August 2017; Accepted 12 October 2017; Published 15 January 2018

Academic Editor: Pierfrancesco Lombardo

Copyright (C) 2018 Heyun Lin et al. This is an open access article distributed under the Creative Commons Attribution License, which permits unrestricted use, distribution, and reproduction in any medium, provided the original work is properly cited.

\begin{abstract}
We provide a complete study on the direction-of-arrival (DOA) estimation of noncircular (NC) signals for uniform linear array (ULA) via Vandermonde constrained parallel factor (PARAFAC) analysis. By exploiting the noncircular property of the signals, we first construct an extended matrix which contains two times sampling number of the received signal. Then, taking the Vandermonde structure of the array manifold matrix into account, the extended matrix can be turned into a tensor model which admits the Vandermonde constrained PARAFAC decomposition. Based on this tensor model, an efficient linear algebra algorithm is applied to obtain the DOA estimation via utilizing the rotational invariance between two submatrices. Compared with some existing algorithms, the proposed method has a better DOA estimation performance. Meanwhile, the proposed method consistently has a higher estimation accuracy and a much lower computational complexity than the trilinear alternating least square- (TALS-) based PARAFAC algorithm. Finally, numerical examples are conducted to demonstrate the effectiveness of the proposed approach in terms of estimation accuracy and computational complexity.
\end{abstract}

\section{Introduction}

Direction-of-arrival (DOA) estimation of signals impinging on an array of sensors is an important and fundamental issue in array signal processing due to its wide applications in wireless communications, geophysics, radar, and so on [1-3]. In this context, many DOA estimators have been developed to solve this problem, such as propagator method (PM) [4], maximum likelihood (ML) methods $[5,6]$, tensor-based method [7], estimation of signal parameters via rotational invariance technique (ESPRIT) algorithm [8], and multiple signal classification (MUSIC) algorithm [9].

Unfortunately, all of the above works [4-9] ignore the characteristics of the incident signals. It has been shown that the accuracy of DOA estimation can be enhanced by taking advantage of the noncircular (NC) property of the impinging signals [10]. Examples of the noncircular signals include binary phase-shift keying (BPSK) and amplitude-modulated (AM) signals which are widely applied in wireless telecommunication systems. While the property of NC signals is utilized, the array aperture is virtually doubled in [10], which yields a better DOA estimation performance than that in [9]. Besides taking the noncircularity of signals into account, the maximum number of sources can potentially exceed the number of array elements. Consequently, solid researches on NC signals for DOA estimation have appeared in many literatures [11-14]. NC ESPRIT algorithm and NC Unitary ESPRIT algorithm for DOA estimation were presented in $[11,12]$, respectively. The work [13] developed a lowcomplexity noncircular rational invariance propagator method (NC-RI-PM) for angle estimation, which is used for uniform linear array (ULA). However, the performance of the NC-RI-PM became worse rapidly in the case of low signal-to-noise ratio (SNR). The authors in [14] utilized the parallel factor (PARAFAC) analysis [15] to acquire the two-dimensional (2D) angle estimation of NC signals for uniform rectangular array (URA). The main drawback in [14] is that it has large computational load and is sensitive to the iterative initial value.

In this paper, we present a Vandermonde constrained PARAFAC method to improve the DOA estimation accuracy by taking advantages of the property of NC signals and the 
array manifold matrix with Vandermonde structure. In order to construct a PARAFAC data model, we first build an extended matrix by concatenating the received data and its conjugated component. Then, the extended data matrix can be formulated as a PARAFAC model with Vandermonde constrained factor matrix. Finally, a closed-form solution can be utilized to acquire the angle information by taking the Vandermonde structure into account.

The contributions of this paper can be summarized as follows: (1) We combine the property of NC signals and the array manifold matrix with Vandermonde structure, based on which a Vandermonde constrained PARAFAC method is proposed to acquire the DOA of NC signals for uniform linear array. (2) Compared with trilinear alternating least square- (TALS-) based PARAFAC method, the proposed method provides a better angle estimation as well as a lower computational complexity. (3) The proposed approach also has a higher estimation accuracy than the ESPRIT method [8], NC ESPRIT method [11], and NC-RI-PM method [13].

The rest of this paper is structured as follows. In Section 2 , we introduce the system model for ULA. Section 3 presents the Vandermonde constrained PARAFAC method for DOA estimation of noncircular signals. The complexity analysis and Cramér-Rao Bound (CRB) of NC signal DOA estimation are given in Section 4. Numerical results are provided in Section 5. Finally, Section 6 concludes the paper.

Notations: scalars, vectors, matrices, and tensors are denoted by lowercase letters, lowercase boldface letters, uppercase boldface letters, and calligraphic letters, respectively. The operators $(\cdot)^{*},(\cdot)^{\mathrm{T}},(\cdot)^{\mathrm{H}},(\cdot)^{\dagger}$, and $\|\cdot\|_{\mathrm{F}}$ denote the conjugate, transpose, conjugate transpose, pseudoinverse, and Frobenius norm, respectively. $\mathbf{I}_{K}$ stands for a $K \times K$ identity matrix. $\operatorname{diag}\{\mathbf{a}\}$ is the diagonal matrix with diagonal elements given by a. $\odot$, ॰, and $\oplus$ represent the Khatri-Rao product, outer product, and Hadamard product, respectively. $r_{\mathbf{A}}$ is the rank of a matrix $\mathbf{A}$.

\section{System Model}

Let us consider a uniform linear array consisting of $M$ omnidirectional sensors. The distance between two adjacent sensors is $d$. As shown in Figure 1, the first sensor is regarded as the reference point for the array, assuming that there are $K$ uncorrelated narrowband far-field signals impinging on this array from distinct directions $\theta_{1}, \ldots, \theta_{K}$. We also assume that the number of sources $K$ is known a priori or has been estimated by the methods shown in $[16,17]$. Under such a scenario, the received signal vector $\mathbf{x}(n) \in \mathbb{C}^{M \times 1}$ can be modeled as

$$
\mathbf{x}(n)=\mathbf{A s}(n)+\mathbf{v}(n)
$$

where

$$
\mathbf{A}=\left[\begin{array}{ccc}
1 & \cdots & 1 \\
\alpha_{1} & \cdots & \alpha_{K} \\
\vdots & \ddots & \vdots \\
\alpha_{1}^{M-1} & \cdots & \alpha_{K}^{M-1}
\end{array}\right]
$$

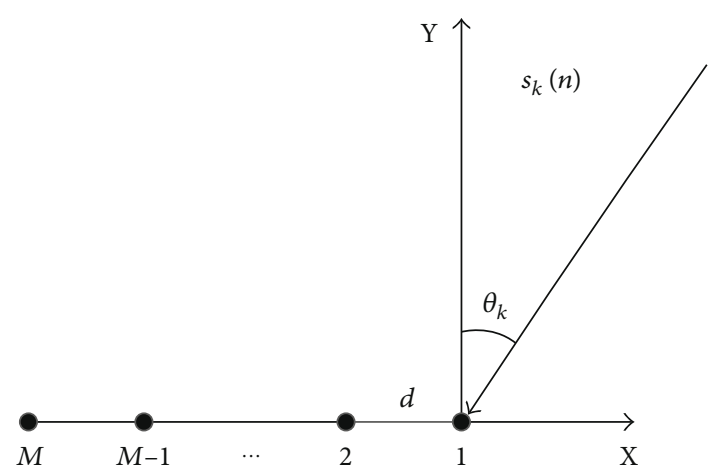

FIGURE 1: Illustration of the array geometry.

is the array manifold matrix with size of $M \times K, \alpha_{k}=$ $e^{-j(2 \pi / \lambda) d \sin \theta_{k}}, \quad k=1, \ldots, K . \lambda$ is the wavelength; $\mathbf{s}(n)=$ $\left[s_{1}(n), \ldots, s_{K}(n)\right]^{\mathrm{T}} \in \mathbb{C}^{K \times 1}$ is the signal vector at snapshot $n$. $\mathbf{v}(n) \in \mathbb{C}^{M \times 1}$ stands for the additive Gaussian noise vector with zero-mean and covariance $\sigma^{2} \mathbf{I}_{M}$. From (2), we know that $\mathbf{A}$ is a Vandermonde matrix with distinct nonzero generators $\alpha_{1}, \ldots, \alpha_{K}$. In this paper, the strictly secondorder noncircular signals [18] are considered. Thus, we have $E[\mathbf{s}(n)]=0_{K \times 1}, E\left[\mathbf{s}(n) \mathbf{s}^{\mathrm{H}}(n)\right] \neq 0_{K \times K}$, and $E\left[\mathbf{s}(n) \mathbf{s}^{\mathrm{T}}(n)\right] \neq 0_{K \times K}$.

It is assumed that the DOAs of the sources are constant in $N$ snapshots. We collect $N$ snapshots corresponding to $\mathbf{x}(n)$; the received signal matrix $\mathbf{X} \in \mathbb{C}^{M \times N}$ is given by

$$
\begin{aligned}
\mathbf{X} & =[\mathbf{x}(1), \ldots, \mathbf{x}(N)] \\
& =\mathbf{A} \mathbf{S}^{\mathrm{T}}+\mathbf{V},
\end{aligned}
$$

where $\mathbf{S}=[\mathbf{s}(1) \ldots \mathbf{s}(N)]^{\mathrm{T}} \in \mathbb{C}^{N \times K}$ stands for the source matrix and $\mathbf{V} \in \mathbb{C}^{M \times N}$ contains the samples of the additive noise. Due to the NC property, we can decompose the source matrix as $\mathbf{S}=\mathbf{S}_{0} \Psi$ [18], where $\mathbf{S}_{0} \in \mathbb{R}^{N \times K}$ is a real-valued matrix, $\quad \boldsymbol{\Psi}=\operatorname{diag}\left\{e^{-j \psi_{1}}, \ldots, e^{-j \psi_{K}}\right\} \in \mathbb{C}^{K \times K}$ is a diagonal matrix, and $\psi_{k}$ is referred to as the phase shift of the $k$ th source. Then (3) can be rewritten as

$$
\mathbf{X}=\mathbf{A} \Psi \mathbf{S}_{0}^{\mathrm{T}}+\mathbf{V}
$$

\section{Estimation of DOA via Vandermonde Constrained PARAFAC Decomposition}

3.1. PARAFAC Model. Since the noncircular signals are considered, we construct the extended data matrix $\overline{\mathbf{X}} \in \mathbb{C}^{2 M \times N}$ as follows $[12,14]$ :

$$
\begin{aligned}
\overline{\mathbf{X}} & =\left[\begin{array}{c}
\mathbf{X} \\
\mathbf{J X}^{*}
\end{array}\right]=\left[\begin{array}{c}
\mathbf{A} \Psi \mathbf{S}_{0}^{\mathrm{T}} \\
\mathbf{J}\left(\mathbf{A} \Psi \mathbf{S}_{0}^{\mathrm{T}}\right)^{*}
\end{array}\right]+\left[\begin{array}{c}
\mathbf{V} \\
\mathbf{J V}^{*}
\end{array}\right] \\
& =\left[\begin{array}{c}
\mathbf{A} \boldsymbol{\Psi} \\
\mathbf{A} \boldsymbol{\Lambda} \boldsymbol{\Psi}^{-1}
\end{array}\right] \mathbf{S}_{0}^{\mathrm{T}}+\mathbf{V}_{1}=\left[\begin{array}{c}
\mathbf{A} D_{1}(\boldsymbol{\Phi}) \\
\mathbf{A} D_{2}(\boldsymbol{\Phi})
\end{array}\right] \mathbf{S}_{0}^{\mathrm{T}}+\mathbf{V}_{1} \\
& =(\boldsymbol{\Phi} \odot \mathbf{A}) \mathbf{S}_{0}^{\mathrm{T}}+\mathbf{V}_{1},
\end{aligned}
$$


where $\mathbf{J} \in \mathbb{R}^{M \times M}$ is the exchange matrix with ones on its antidiagonal and zeros elsewhere. $\mathbf{V}_{1}$ is the extended noise matrix. $\boldsymbol{\Lambda}=\operatorname{diag}\left(e^{j(2 \pi / \lambda)(M-1) d \sin \theta_{1}}, \ldots\right.$, $\left.e^{j(2 \pi / \lambda)(M-1) d \sin \theta_{K}}\right) \in \mathbb{C}^{K \times K}$, and

$$
\boldsymbol{\Phi}=\left[\begin{array}{ccc}
e^{-j \psi_{1}} & , \ldots, & e^{-j \psi_{K}} \\
e^{j\left[\frac{2 \pi}{\lambda}(M-1) d \sin \theta_{1}+\psi_{1}\right]} & , \ldots, & \left.e^{j\left[\frac{2 \pi}{\lambda}(M-1) d \sin \theta_{K}+\psi_{K}\right.}\right]
\end{array}\right]
$$

is a $2 \times K$ matrix. $D_{i}\{\boldsymbol{\Phi}\}$ is the diagonal matrix with diagonal elements given by the $i$ th row of $\boldsymbol{\Phi}$. According to Harshman [15], the signal part of (5) can be seen as a matrix representation of a third-order PARAFAC model $\mathcal{X} \in \mathbb{C}^{M \times N \times P}$, whose $(m, n, p)-$ th element is given by

$$
\bar{x}_{m, n, p}=\sum_{k=1}^{K} a_{m, k} s_{0 n, k} \phi_{p, k}
$$

where $m=1, \ldots, M, n=1, \ldots, N$, and $p=1,2 . \bar{x}_{m, n, p}$ represents the typical element of the tensor $\mathcal{X} . a_{m, k}$ denotes the $(m, k)$ - th element of $\mathbf{A}$, and similarly for the others. Using the reshaping operations, we can obtain the other two equivalent matrix representations of $\mathcal{X}$ as follows:

$$
\begin{aligned}
& \overline{\mathbf{Y}}=\left(\mathbf{A} \odot \mathbf{S}_{0}\right) \boldsymbol{\Phi}^{\mathrm{T}}+\mathbf{V}_{2} \in \mathbb{C}^{M N \times 2}, \\
& \overline{\mathbf{Z}}=\left(\mathbf{S}_{0} \odot \boldsymbol{\Phi}\right) \mathbf{A}^{\mathrm{T}}+\mathbf{V}_{3} \in \mathbb{C}^{2 N \times M} .
\end{aligned}
$$

Till now, we successfully link the expanded data matrix with a tensor model which satisfies the PARAFAC decomposition. According to (5), the PARAFAC decomposition can be accomplished by solving the following unconstrained optimization problem.

$$
\min _{\mathbf{A}, \mathbf{S}_{0}, \boldsymbol{\Phi}}\left\|\chi-\sum_{k=1}^{K} \mathbf{a}_{k} \circ \mathbf{s}_{0 k} \circ \boldsymbol{\phi}_{k}\right\|_{\mathrm{F}}^{2},
$$

where $\mathbf{a}_{k}, \mathbf{s}_{0 k}$, and $\phi_{k}$ represent the $k$ th columns of the matrices $\mathbf{A}, \mathbf{S}_{0}$, and $\boldsymbol{\Phi}$, respectively. This is most often done by means of the TALS method. As its programming simplicity, the TALS algorithm has been successfully employed to estimate the parameters of several tensor models $[7,19,20]$. The basic idea of the TALS algorithm for fitting the proposed PARAFAC model is stated as follows:

With the consideration of (5), while $\mathbf{A}$ and $\boldsymbol{\Phi}$ are fixed, we update $\mathbf{S}_{0}$ using the following least squares (LS) fitting:

$$
\begin{aligned}
\widehat{\mathbf{S}}_{0} & =\underset{\mathbf{S}_{0}}{\arg \min }\left\|\overline{\mathbf{X}}-(\boldsymbol{\Phi} \odot \mathbf{A}) \mathbf{S}_{0}^{\mathrm{T}}\right\|_{\mathrm{F}} \\
& =\left((\boldsymbol{\Phi} \odot \mathbf{A})^{\dagger} \overline{\mathbf{X}}\right)^{\mathrm{T}},
\end{aligned}
$$

where $\widehat{\mathbf{S}}_{0}$ stands for the estimation of $\mathbf{S}_{0}$. In the same way, while $\mathbf{A}$ and $\mathbf{S}_{0}$ are fixed, $\boldsymbol{\Phi}$ can be updated by the following LS fitting:

$$
\begin{aligned}
\widehat{\boldsymbol{\Phi}} & =\underset{\boldsymbol{\Phi}}{\arg \min }\left\|\overline{\mathbf{Y}}-\left(\mathbf{A} \odot \mathbf{S}_{0}\right) \boldsymbol{\Phi}^{\mathrm{T}}\right\|_{\mathrm{F}} \\
& =\left(\left(\mathbf{A} \odot \mathbf{S}_{0}\right)^{\dagger} \overline{\mathbf{Y}}\right)^{\mathrm{T}},
\end{aligned}
$$

where $\widehat{\boldsymbol{\Phi}}$ stands for the estimation of $\boldsymbol{\Phi}$. Similarly, we obtain an updated $\mathbf{A}$ via LS fitting as

$$
\begin{aligned}
\widehat{\mathbf{A}} & =\underset{\mathbf{A}}{\arg \min }\left\|\overline{\mathbf{Z}}-\left(\mathbf{S}_{0} \odot \boldsymbol{\Phi}\right) \mathbf{A}^{\mathrm{T}}\right\|_{\mathrm{F}} \\
& =\left(\left(\mathbf{S}_{0} \odot \boldsymbol{\Phi}\right)^{\dagger} \overline{\mathbf{Z}}\right)^{\mathrm{T}},
\end{aligned}
$$

where $\widehat{\mathbf{A}}$ stands for the estimation of $\mathbf{A}$. Repeat updating process of the three matrices until convergence, and then the estimation matrices $\widehat{\mathbf{A}}, \widehat{\mathbf{S}}_{0}$, and $\widehat{\boldsymbol{\Phi}}$ can be obtained. It is well known that the TALS algorithm is sensitive to the initial value as well as the array manifold, which may cause the TALS algorithm converging slowly. In the next subsection, a more efficient algorithm is applied to obtain the estimation of DOA for noncircular signals.

3.2. DOA Estimation with Vandermonde Constrained PARAFAC Method. From the previous analysis, we know that the matrix $\mathbf{A}$ is a Vandermonde matrix with distinct nonzero generators $\alpha_{1}, \ldots, \alpha_{K}$. Therefore, by taking the Vandermonde factor into account, the model $\mathcal{X}$ can be seen as a tensor which admits the PARAFAC decomposition with Vandermonde constraint. And then, a more efficient algorithm is applied to fitting the PARAFAC model with Vandermonde constraint.

According to (6), we know that $r_{\Phi}=\min (2, K)$. If $K>2$, we can conclude that the matrix $\Phi$ does not have fullcolumn rank, which implies that $r_{\Phi}$ is less than $K$. Then, the rank of $\overline{\mathbf{Y}}$ is also less than $K$. Consequently, the spatial smoothing technique can be used to handle with this problem [21]. The detailed process of spatial smoothing is presented as follows:

Denote $\overline{\mathbf{Y}}^{\left(l_{2}\right)}$ as the $l_{2}$ th subarray of $\overline{\mathbf{Y}}$ such that $\overline{\mathbf{Y}}^{\left(l_{2}\right)}=\overline{\mathbf{Y}}$ $\left(N\left(l_{2}-1\right)+1: N\left(L_{1}+l_{2}-1\right),:\right), l_{2}=1, \ldots, L_{2}$, where $L_{2}$ is the smoothing factor. The integers $L_{1}$ and $L_{2}$ must be chosen such that $M=L_{1}+L_{2}-1$. Taking the advantage of the Vandermonde structure of $\mathbf{A}$, we have

$$
\overline{\mathbf{Y}}^{\left(l_{2}\right)}=\left[\begin{array}{c}
\mathbf{S}_{0} D_{l_{2}}(\mathbf{A}) \\
\mathbf{S}_{0} D_{l_{2}+1}(\mathbf{A}) \\
\vdots \\
\mathbf{S}_{0} D_{l_{2}+L_{1}-1}(\mathbf{A})
\end{array}\right] \boldsymbol{\Phi}^{\mathrm{T}}=\left[\begin{array}{c}
\mathbf{S}_{0} D_{1}(\mathbf{A}) \\
\mathbf{S}_{0} D_{2}(\mathbf{A}) \\
\vdots \\
\mathbf{S}_{0} D_{L_{1}}(\mathbf{A})
\end{array}\right] \boldsymbol{\Omega}^{l_{2}-1} \boldsymbol{\Phi}^{\mathrm{T}},
$$

where $\boldsymbol{\Omega}=\operatorname{diag}\left(e^{j(2 \pi / \lambda) d \sin \theta_{1}}, \ldots, e^{j(2 \pi / \lambda) d \sin \theta_{K}}\right) \in \mathbb{C}^{K \times K}$. Then, denote $\tilde{\mathbf{Y}}=\left[\begin{array}{lll}\overline{\mathbf{Y}}^{(1)} & \ldots & \overline{\mathbf{Y}}^{\left(L_{2}\right)}\end{array}\right] \in \mathbb{C}^{N L_{1} \times 2 L_{2}}$, we can obtain 


$$
\begin{aligned}
\tilde{\mathbf{Y}} & =\left[\begin{array}{lll}
\overline{\mathbf{Y}}^{(1)} & \ldots & \overline{\mathbf{Y}}^{\left(L_{2}\right)}
\end{array}\right] \\
& =\left[\begin{array}{c}
\mathbf{S}_{0} D_{1}(\mathbf{A}) \\
\mathbf{S}_{0} D_{2}(\mathbf{A}) \\
\vdots \\
\mathbf{S}_{0} D_{L_{1}}(\mathbf{A})
\end{array}\right]\left[\begin{array}{llll}
\boldsymbol{\Phi}^{\mathrm{T}} & \boldsymbol{\Omega} \boldsymbol{\Phi}^{\mathrm{T}} & \cdots & \boldsymbol{\Omega}^{L_{2}-1} \boldsymbol{\Phi}^{\mathrm{T}}
\end{array}\right] \\
& =\left(\mathbf{A}^{\left(L_{1}\right)} \odot \mathbf{S}_{0}\right)\left[\begin{array}{llll}
\boldsymbol{\Phi}^{\mathrm{T}} & \boldsymbol{\Omega} \boldsymbol{\Phi}^{\mathrm{T}} & \cdots & \boldsymbol{\Omega}^{L_{2}-1} \boldsymbol{\Phi}^{\mathrm{T}}
\end{array}\right],
\end{aligned}
$$

where $\mathbf{A}^{\left(L_{1}\right)} \in \mathbb{C}^{L_{1} \times K}$ denotes the first $L_{1}$ rows of $\mathbf{A}$. Note that

$$
\begin{aligned}
{\left[\begin{array}{llll}
\boldsymbol{\Phi}^{\mathrm{T}} & \boldsymbol{\Omega} \boldsymbol{\Phi}^{\mathrm{T}} & \cdots & \boldsymbol{\Omega}^{L_{2}-1} \boldsymbol{\Phi}^{\mathrm{T}}
\end{array}\right]^{\mathrm{T}}=\left[\begin{array}{c}
\boldsymbol{\Phi} D_{1}(\mathbf{A}) \\
\boldsymbol{\Phi} D_{2}(\mathbf{A}) \\
\vdots \\
\boldsymbol{\Phi} D_{L_{2}}(\mathbf{A})
\end{array}\right] } \\
=\left(\mathbf{A}^{\left(L_{2}\right)} \odot \boldsymbol{\Phi}\right),
\end{aligned}
$$

where $\mathbf{A}^{\left(L_{2}\right)} \in \mathbb{C}^{L_{2} \times K}$ denotes the first $L_{2}$ rows of $\mathbf{A}$.

Therefore, (14) can be rewritten as

$$
\tilde{\mathbf{Y}}=\left(\mathbf{A}^{\left(L_{1}\right)} \odot \mathbf{S}_{0}\right)\left(\mathbf{A}^{\left(L_{2}\right)} \odot \boldsymbol{\Phi}\right)^{\mathrm{T}} .
$$

In this way, the matrix representation $\overline{\mathbf{Y}}$ of the tensor $\mathcal{X}$, which involves a rank-deficient matrix $\Phi$ (while $K>2$ ), is replaced by a matrix representation $\tilde{\mathbf{Y}}$ which only involves two full-column rank matrices $\mathbf{A}^{\left(L_{1}\right)} \odot \mathbf{S}_{0}$ and $\mathbf{A}^{\left(L_{2}\right)} \odot \boldsymbol{\Phi}$. The matrix $\tilde{\mathbf{Y}}$ can be seen as a matrix representation of a constrained PARAFAC decomposition model [22-24] with three factors $\mathbf{A}^{\left(L_{1}\right)}, \mathbf{S}_{0}$ and $\mathbf{A}^{\left(L_{2}\right)} \odot \boldsymbol{\Phi}$. When the matrix $\tilde{\mathbf{Y}}$ is obtained, a more efficient algorithm can be used to fit the Vandermonde constrained PARAFAC model [23, 24].

While the matrices $\mathbf{A}^{\left(L_{1}\right)} \odot \mathbf{S}_{0}$ and $\mathbf{A}^{\left(L_{2}\right)} \odot \boldsymbol{\Phi}$ have fullcolumn rank, we perform the compact singular value decomposition (SVD) of $\tilde{\mathbf{Y}}$, which is given by $\tilde{\mathbf{Y}} \approx \mathbf{U} \mathbf{\Sigma} \mathbf{V}^{\mathrm{H}}$, where $\Sigma \in \mathbb{C}^{K \times K}$ is a diagonal matrix consisting of the largest $K$ nonzero singular values. $\mathbf{U} \in \mathbb{C}^{L_{1} N \times K}$ and $\mathbf{V} \in \mathbb{C}^{2 L_{2} \times K}$ are matrices composed of left and right singular vectors associated with the largest $K$ nonzero singular values, respectively. Since the factor matrices $\mathbf{A}^{\left(L_{1}\right)} \odot \mathbf{S}_{0}$ and $\mathbf{A}^{\left(L_{2}\right)} \odot \boldsymbol{\Phi}$ have fullcolumn rank, we can conclude that the subspace spanned by the column vectors of $\mathbf{U}$ is the same as the subspace spanned by the column vectors of the matrix $\mathbf{A}^{\left(L_{1}\right)} \odot \mathbf{S}_{0}$, so we have

$$
\operatorname{span}\left(\mathbf{A}^{\left(L_{1}\right)} \odot \mathbf{S}_{0}\right)=\operatorname{span}(\mathbf{U}) .
$$

Therefore, there exists a unique nonsingular matrix $\mathbf{M} \in \mathbb{C}^{K \times K}$ such that

$$
\mathbf{U M}=\mathbf{A}^{\left(L_{1}\right)} \odot \mathbf{S}_{0} .
$$

Using the inherent Vandermonde structure of $\mathbf{A}^{\left(L_{1}\right)}$, we define the following two submatrices:

$$
\begin{gathered}
\mathbf{U}_{1} \mathbf{M}=\underline{\mathbf{A}}^{\left(L_{1}\right)} \odot \mathbf{S}_{0}=\left[\begin{array}{c}
\mathbf{S}_{0} D_{1}(\mathbf{A}) \\
\mathbf{S}_{0} D_{2}(\mathbf{A}) \\
\vdots \\
\mathbf{S}_{0} D_{L_{1}-1}(\mathbf{A})
\end{array}\right], \\
\mathbf{U}_{2} \mathbf{M}=\overline{\mathbf{A}}^{\left(L_{1}\right)} \odot \mathbf{S}_{0}=\left[\begin{array}{c}
\mathbf{S}_{0} D_{2}(\mathbf{A}) \\
\mathbf{S}_{0} D_{3}(\mathbf{A}) \\
\vdots \\
\mathbf{S}_{0} D_{L_{1}}(\mathbf{A})
\end{array}\right],
\end{gathered}
$$

where $\mathbf{U}_{1}=\mathbf{U}\left(1:\left(L_{1}-1\right) N, 1: K\right)$ and $\mathbf{U}_{2}=\mathbf{U}\left(N+1: L_{1} N\right.$, $1: K)$, and the relationship between $\mathbf{A}^{\left(L_{1}\right)}, \underline{\mathbf{A}}^{\left(L_{1}\right)}$, and $\overline{\mathbf{A}}^{\left(L_{1}\right)}$ is that

$$
\mathbf{A}^{\left(L_{1}\right)}=\left[\begin{array}{c}
\underline{\mathbf{A}}^{\left(L_{1}\right)} \\
\text { last row }
\end{array}\right]=\left[\begin{array}{c}
\text { first row } \\
\overline{\mathbf{A}}^{\left(L_{1}\right)}
\end{array}\right] .
$$

Therefore, there exists a diagonal matrix $\mathbf{Z} \in \mathbb{C}^{K \times K}$ such that

$$
\left(\underline{\mathbf{A}}^{\left(L_{1}\right)} \odot \mathbf{S}_{0}\right) \mathbf{Z}=\left(\overline{\mathbf{A}}^{\left(L_{1}\right)} \odot \mathbf{S}_{0}\right),
$$

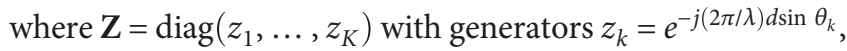
$k=1, \ldots, K$.

According to (19), (20), (21) and (22), we can obtain that

$$
\mathbf{U}_{2} \mathbf{M}=\mathbf{U}_{1} \mathbf{M Z} \text {. }
$$

From (23), we have $\mathbf{U}_{2}=\mathbf{U}_{1} \mathbf{M} \mathbf{Z} \mathbf{M}^{-1}=\mathbf{U}_{1} \widehat{\mathbf{Z}}$, where $\widehat{\mathbf{Z}}=$ $\mathbf{M Z M}^{-1}$. Assuming that the matrix $\underline{\mathbf{A}}^{\left(L_{1}\right)} \odot \mathbf{S}_{0}$ has fullcolumn rank, we can conclude that the matrices $\mathbf{U}_{1}$ and $\mathbf{U}_{2}$ have full-column rank and $\widehat{\mathbf{Z}}$ can be rewritten as

$$
\widehat{\mathbf{Z}}=\mathbf{U}_{1}^{\dagger} \mathbf{U}_{2}=\mathbf{M Z M}^{-1} \text {. }
$$

Therefore, the generators $z_{k}, k=1, \ldots, K$, can be obtained from the eigenvalue decomposition (EVD) of $\mathbf{U}_{1}^{\dagger}$ $\mathbf{U}_{2}$. Then, the DOA of the $k$ th source $\theta_{k}$ can be recovered via

$$
\widehat{\theta}_{k}=\arcsin \left(\frac{-\lambda \operatorname{angle}\left(z_{k}\right)}{2 \pi d}\right) \quad k=1, \ldots, K,
$$

where $\widehat{\theta}_{k}$ stands for the estimation of $\theta_{k}$ and angle $(\cdot)$ is used for obtaining the phase. The proposed Vandermonde constrained PARAFAC algorithm for NC signals DOA estimation is presented in Algorithm 1.

Remark 1. The proposed method in this paper is developed for noncircular signals. Therefore, we assume that the circular signals are not present in this paper. When the mixed noncircular and circular signals are considered, the DOAs can be estimated by the algorithms reported in $[25,26]$.

3.3. Identifiabilitity Analysis. The most remarkable characteristic of the PARAFAC decomposition is its essential uniqueness property, which makes PARAFAC decomposition a key tool for signal separation. In this subsection, 
Step 1. Construct the tensor model $\mathcal{X}$ with the matrix representation $\overline{\mathbf{Y}}=\left(\mathbf{A} \odot \mathbf{S}_{0}\right) \boldsymbol{\Phi}^{\mathrm{T}}$ by exploiting the property of NC signals.

Step 2. Choose the pair $\left(L_{1}, L_{2}\right)$ subject to $L_{1}+L_{2}=M+1$ and construct the matrix representation $\tilde{\mathbf{Y}}=\left(\mathbf{A}^{\left(L_{1}\right)} \odot \mathbf{S}_{0}\right)\left(\mathbf{A}^{\left(L_{2}\right)} \odot \boldsymbol{\Phi}\right)^{\mathrm{T}}$ via spatial smoothing.

Step 3. Perform the compact SVD of $\tilde{\mathbf{Y}}: \tilde{\mathbf{Y}} \approx \mathbf{U} \Sigma \mathbf{V}^{\mathrm{H}}$.

Step 4. Construct $\mathbf{U}_{1}=\mathbf{U}\left(1:\left(L_{1}-1\right) N, 1: K\right), \mathbf{U}_{2}=\mathbf{U}\left(N+1: L_{1} N, 1: K\right)$.

Step 5. Perform the EVD of $\mathbf{U}_{1}^{\dagger} \mathbf{U}_{2}: \mathbf{U}_{1}^{\dagger} \mathbf{U}_{2}=\mathbf{M Z M}^{-1}, \mathbf{Z}=\operatorname{diag}\left(z_{1}, \ldots, z_{K}\right)$ with generators $z_{k}=e^{-j(2 \pi / \lambda) d \sin \theta_{k}}, k=1, \ldots, K$.

Step 6. Compute $\widehat{\theta}_{k}=\arcsin \left(-\lambda \operatorname{angle}\left(z_{k}\right) / 2 \pi d\right), k=1, \ldots, K$.

Algorithm 1: The Vandermonde constrained PARAFAC algorithm for NC signals DOA estimation.

we discuss the identifiabilitity condition for the proposed PARAFAC method.

Theorem $1[7,15,27,28]$. Let $\mathcal{X} \in \mathbb{C}^{M \times N \times 2}$ be a tensor with matrix representation $\overline{\mathbf{Y}}=\left(\mathbf{A} \odot \mathbf{S}_{0}\right) \Phi^{\mathrm{T}}$, where $\mathbf{A} \in \mathbb{C}^{M \times K}, \mathbf{S}_{0}$ $\in \mathbb{R}^{N \times K}$, and $\Phi \in \mathbb{C}^{2 \times K}$, if

$$
k_{\mathrm{A}}+k_{\mathbf{S}_{0}}+k_{\Phi} \geq 2 K+2 \text {, }
$$

then the three factor matrices $\mathbf{A}, \mathbf{S}_{0}$, and $\boldsymbol{\Phi}$ are unique up to permutation and scaling of columns, meaning that any other triple $\left(\widehat{\mathbf{A}}, \widehat{\mathbf{S}}_{0}, \widehat{\Phi}\right)$ is related to $\left(\mathbf{A}, \mathbf{S}_{0}, \boldsymbol{\Phi}\right)$ via

$$
\widehat{\mathrm{A}}=\mathrm{A} \Pi \Delta_{1}, \widehat{\mathrm{S}}_{0}=\mathrm{S}_{0} \Pi \Delta_{2}, \widehat{\Phi}=\Phi \Pi \Delta_{3},
$$

where $k_{\mathrm{A}}, k_{\mathrm{S}_{0}}$, and $k_{\Phi}$ are the $k$ - ranks $[27,28]$ of the matrices $\mathbf{A}, \mathbf{S}_{0}$, and $\boldsymbol{\Phi}$, respectively. $\boldsymbol{\Pi}$ is a permutation matrix. $\boldsymbol{\Delta}_{i}$, $i=1, \ldots, 3$, are diagonal scaling matrices satisfying $\Delta_{1} \Delta_{2}$ $\Delta_{3}=\mathbf{I}_{K}$.

If the three factor matrices have full rank, then condition (26) becomes

$$
\min (M, K)+\min (N, K)+\min (2, K) \geq 2 K+2 .
$$

In this paper, we assume $N \geq K, K \geq 2$, then the identifiable condition (28) becomes $M \geq K$. Therefore, the maximum number of sources that can be handled by the PARAFAC algorithm is no more than $M$.

When $\mathcal{X} \in \mathbb{C}^{M \times N \times P}$ is a tensor that admits the Vandermonde constrained PARAFAC decomposition, we have the following uniqueness result.

Theorem $2[23,24]$. Let $\mathcal{X} \in \mathbb{C}^{M \times N \times 2}$ be a tensor with matrix representation $\overline{\mathbf{Y}}=\left(\mathbf{A} \odot \mathbf{S}_{0}\right) \boldsymbol{\Phi}^{\mathrm{T}}$, where $\mathbf{A} \in \mathbb{C}^{M \times K}$ is a Vandermonde matrix with distinct generators, $\mathbf{S}_{0} \in$ $\mathbb{R}^{N \times K}$ and $\Phi \in \mathbb{C}^{2 \times K}$. Consider the matrix representation $\tilde{\mathbf{Y}}=\left(\mathbf{A}^{\left(L_{1}\right)} \odot \mathbf{S}_{0}\right)\left(\mathbf{A}^{\left(L_{2}\right)} \odot \boldsymbol{\Phi}\right)^{\mathrm{T}}$ with $L_{1}+L_{2}=M+1$. If

$$
\begin{aligned}
& r\left(\left(\underline{\mathbf{A}}^{\left(L_{1}\right)} \odot \mathbf{S}_{0}\right)\right)=K, \\
& r\left(\left(\mathbf{A}^{\left(L_{2}\right)} \odot \boldsymbol{\Phi}\right)\right)=K,
\end{aligned}
$$

for some $L_{1}+L_{2}=M+1$, then $K=r(\mathcal{X})$, and the Vandermonde constrained PARAFAC decomposition of $\mathcal{X}$ is unique. Generically, condition (29) is satisfied if and only if

$$
\min \left(\left(L_{1}-1\right) N, 2 L_{2}\right) \geq K
$$

When we assume $N \geq M$ and $L_{1} \geq 2$, the condition (30) becomes $2 L_{2} \geq K$. Therefore, the maximum number of sources that can be identified by the Vandermonde constrained PARAFAC algorithm is $2 L_{2}$. If $2 L_{2}>M$, the Vandermonde constrained PARAFAC method can identify more sources than the PARAFAC method under the same conditions. Notably, the scaling does not involve the Vandermonde factor matrix in a PARAFAC model with Vandermode constraint. This means that the estimated matrices $\tilde{\mathbf{A}}, \tilde{\mathbf{S}}_{0}$, and $\tilde{\boldsymbol{\Phi}}$ are related to $\mathbf{A}, \mathbf{S}_{0}$, and $\Phi$ via

$$
\tilde{\mathbf{A}}=\mathbf{A} \Pi, \tilde{\mathbf{S}}_{0}=\mathbf{S}_{0} \Pi \tilde{\Delta}_{2}, \tilde{\Phi}=\Phi \Pi \tilde{\Delta}_{3},
$$

where $\tilde{\Delta}_{j}, j=2,3$, are diagonal scaling matrices satisfying $\tilde{\Delta}_{2} \tilde{\Delta}_{3}=\mathbf{I}_{K}$.

Remark 2. The proposed method can be seen as a generalized ESPRIT method. In line with the experiments for ESPRIT in $[29,30]$, we can choose the pair $\left(L_{1}, L_{2}\right)$ such that the dimensions of the matrices $\mathbf{A}^{\left(L_{1}\right)} \odot \mathbf{S}_{0}$ and $\mathbf{A}^{\left(L_{2}\right)} \odot \boldsymbol{\Phi}$ are close, with the inequality $\min \left(\left(L_{1}-1\right) N, 2 L_{2}\right) \geq K$ satisfied. According to $[29,30]$, we know that such a direct method will yield a very good estimation result. Therefore, in order to reduce the computational complexity, $L_{1}$ is fixed to 3 and $L_{2}=M-2$ in this paper. Although it may not yield the best estimation result with the parameters being set as $L_{1}=3$ and $L_{2}=M-2$, a good estimation result can be still achieved. Note that simulations in Section 5 show that the DOA performance of such a direct approach still outperforms than other methods.

\section{Performance Analysis}

4.1. Computational Complexity Analysis. In this subsection, we discuss the computational complexity of the proposed Vandermonde constrained PARAFAC method. The main computational tasks of the Vandermonde constrained PARAFAC method are computing the compact SVD of $\tilde{\mathbf{Y}}$ which requires $\mathcal{O}\left(2 N L_{1} L_{2} K\right)$ [31], pseudoinverse operation of matrix $\mathbf{U}_{1}$ which requires $\mathcal{O}\left(2\left(L_{1}-1\right) N K^{2}\right)$, and the EVD of $\mathbf{U}_{1}^{\dagger} \mathbf{U}_{2}$ which requires $\mathcal{O}\left(K^{3}\right)$. Therefore, the main computational complexity of the proposed Vandermonde constrained PARAFAC method is $\mathcal{O}\left(2 N L_{1} L_{2} K+2\left(L_{1}-1\right)\right.$ 
TABLE 1: Computational complexity of the proposed algorithm and TALS-based PARAFAC.

\begin{tabular}{lc}
\hline Algorithm & Computational complexity \\
\hline The proposed method & $\mathcal{O}\left(2 N L_{1} L_{2} K+2\left(L_{1}-1\right) N K^{2}+K^{3}\right)$ \\
TALS-based PARAFAC & $\mathcal{O}\left(\left(K^{3}+2 N M K\right) n_{1}\right)$ \\
\hline
\end{tabular}

$N K^{2}+K^{3}$ ). The TALS-based PARAFAC method is also illustrated for comparison. The computational cost of TALS-based PARAFAC method is $\mathcal{O}\left(\left(K^{3}+2 N M K\right) n_{1}\right)$ [32], where $n_{1}$ is the number of iterations. We summarize the main computational complexity of the proposed Vandermonde constrained PARAFAC method and the TALS-based PARAFAC method in Table 1.

4.2. Cramér-Rao Bound (CRB). From [33], we can derive the Cramér-Rao Bound (CRB) of noncircular signal DOA estimation for ULA as follows:

$$
\mathrm{CRB}=\frac{\sigma^{2}}{2 N}\left\{\operatorname{Re}\left[\mathbf{D}^{\mathrm{H}} \boldsymbol{\Pi}_{\mathbf{B}}^{\perp} \mathbf{D} \oplus \widehat{\mathbf{P}}^{\mathrm{T}}\right]\right\}^{-1}
$$

where $\sigma^{2}$ is the noise power, $\mathbf{B}=\boldsymbol{\Phi} \odot \mathbf{A}=\left[\mathbf{b}_{1}, \ldots, \mathbf{b}_{K}\right]$, $\boldsymbol{\Pi}_{\mathbf{B}}^{\perp}=\mathbf{I}-\mathbf{B}\left(\mathbf{B}^{\mathrm{H}} \mathbf{B}\right)^{-1} \mathbf{B}^{\mathrm{H}}, \quad \widehat{\mathbf{P}}=(1 / N) \sum_{n=1}^{N} \overline{\mathbf{s}}_{0}(n) \overline{\mathbf{s}}_{0}^{\mathrm{H}}(n), \quad \overline{\mathbf{s}}_{0}(n)=$ $\left[\mathbf{s}_{0}(n) \mathbf{s}_{0}(n)\right]^{\mathrm{T}}, \mathbf{s}_{0}(n)$ is the $n$th row of $\mathbf{S}_{0}, \mathbf{D}=\left[\mathbf{d}_{1 \theta}, \ldots, \mathbf{d}_{K \theta}\right.$, $\left.\mathbf{d}_{1 \psi}, \ldots, \mathbf{d}_{K \psi}\right], \quad \mathbf{d}_{k \theta}=\partial \mathbf{b}_{k} / \partial \theta_{k}$, and $\mathbf{d}_{k \psi}=\partial \mathbf{b}_{k} / \partial \psi_{k}$ with $\mathbf{b}_{k}$ being the $k$ th column of $\mathbf{B}$.

Remark 3. Since the proposed Vandermonde constrained PARAFAC solution can be seen as a generalized ESPRIT method, hence it could be better optimized by weighting subspace fitting methods such as $[34,35]$.

\section{Simulation Examples}

The simulations are presented to evaluate the DOA estimation performance of the proposed method in this section. The root mean square error (RMSE) is used to evaluate the DOA estimation performance, which is defined as

$$
\mathrm{RMSE}=\frac{1}{K} \sum_{k=1}^{K} \sqrt{\frac{1}{500} \sum_{q=1}^{500}\left(\hat{\theta}_{k, q}-\theta_{k}\right)^{2}}
$$

where $\hat{\theta}_{k, q}$ is the estimated values of $\theta_{k}$ in the $q$ th trial, $q=1, \ldots, 500$. $K$ is the number of sources.

Figure 2 shows the DOA estimation result of the proposed Vandermonde constrained PARAFAC method. In this simulation, we set $M=8, N=300, \mathrm{SNR}=5 \mathrm{~dB}$. 30 Monte Carlo trials are carried out. Moreover, we assume that there exist $K=4$ noncircular signals locating at angles $\left(\theta_{1}, \theta_{2}, \theta_{3}\right.$, $\left.\theta_{4}\right)=\left(10^{\circ}, 20^{\circ}, 30^{\circ}, 40^{\circ}\right)$, and the noncircular phases are set as $\left(\psi_{1}, \psi_{2}, \psi_{3}, \psi_{4}\right)=\left(20^{\circ}, 30^{\circ}, 50^{\circ}, 60^{\circ}\right)$. It can be seen from Figure 2 that our Vandermonde constrained PARAFAC method has a good DOA estimation performance even at a

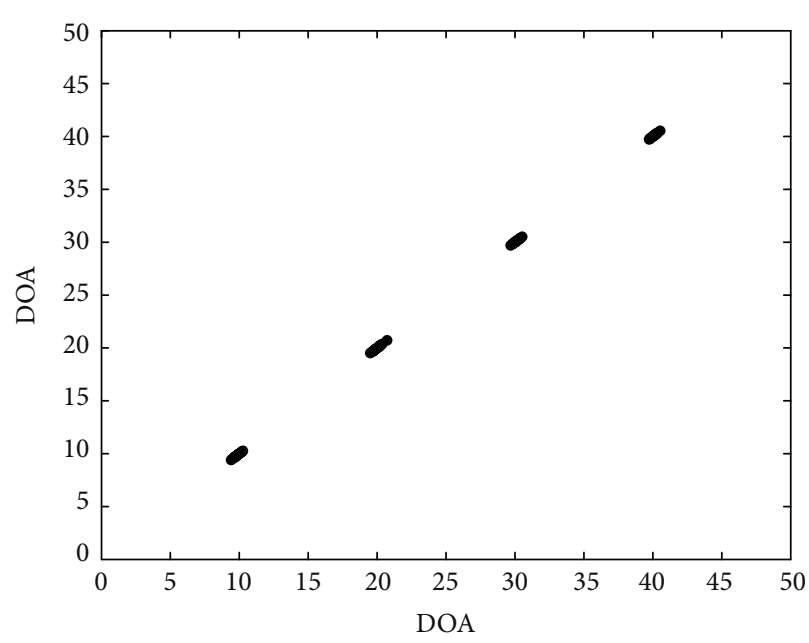

FIgURe 2: Angle estimation result for 30 independent trials with $\mathrm{SNR}=5 \mathrm{~dB}$.

low SNR level. Therefore, the effectiveness of the proposed method is verified.

Figure 3 depicts the DOA estimation result of the proposed Vandermonde constrained PARAFAC method for close sources. In this simulation, we assume $M=8, N=$ $300, \mathrm{SNR}=15 \mathrm{~dB}, K=4,\left(\theta_{1}, \theta_{2}, \theta_{3}, \theta_{4}\right)=\left(10^{\circ}, 20^{\circ}, 22^{\circ}, 40^{\circ}\right)$, and $\left(\psi_{1}, \psi_{2}, \psi_{3}, \psi_{4}\right)=\left(20^{\circ}, 30^{\circ}, 50^{\circ}, 60^{\circ}\right)$. The number of the Monte Carlo trials is set to 30. From Figure 3, it can be observed that the proposed Vandermonde constrained PARAFAC method can separate close sources effectively. Moreover, Figures 2 and 3 present that the DOA estimation performance could be improved with SNR increasing.

Figure 4 presents the RMSE of the proposed Vandermonde constrained PARAFAC method versus SNR. We assume that $K=4,\left(\theta_{1}, \theta_{2}, \theta_{3}, \theta_{4}\right)=\left(5^{\circ}, 15^{\circ}, 45^{\circ}, 60^{\circ}\right),\left(\psi_{1}\right.$, $\left.\psi_{2}, \psi_{3}, \psi_{4}\right)=\left(10^{\circ}, 20^{\circ}, 30^{\circ}, 40^{\circ}\right), M=8$, and $N=200$. The TALS-based PARAFAC algorithm, ESPRIT algorithm in [8], NC-ESPRIT algorithm in [11], NC-RI-PM algorithm in [13], and the CRB are also illustrated for comparison in Figure 4. From Figure 4, we can see that the Vandermonde constrained PARAFAC method can achieve a better estimation performance than ESPRIT, NC-ESPRIT, and NC-RIPM. Notably, by taking the Vandermonde structure into account, our Vandermonde constrained PARAFAC method outperforms the TALS-based PARAFAC approach.

The bias and variance of the estimator versus SNR are shown in Figures 5 and 6 , respectively. We consider there exist $K=4$ noncircular signals locating at the angles of $\left(\theta_{1}, \theta_{2}, \theta_{3}, \theta_{4}\right)=\left(10^{\circ}, 20^{\circ}, 40^{\circ}, 60^{\circ}\right)$, and the noncircular phases are set as $\left(\psi_{1}, \psi_{2}, \psi_{3}, \psi_{4}\right)=\left(20^{\circ}, 30^{\circ}, 40^{\circ}, 50^{\circ}\right)$. The number of antennas is set as $M=8$, the snapshot $N$ is fixed to 200, while SNR is varied from 0 to $30 \mathrm{~dB}$. As expected, we can see from Figures 5 and 6 that the bias and variance of the estimator decrease as SNR increases, respectively.

Table 2 shows the comparison of average run time (s) between Vandermonde constrained PARAFAC method 


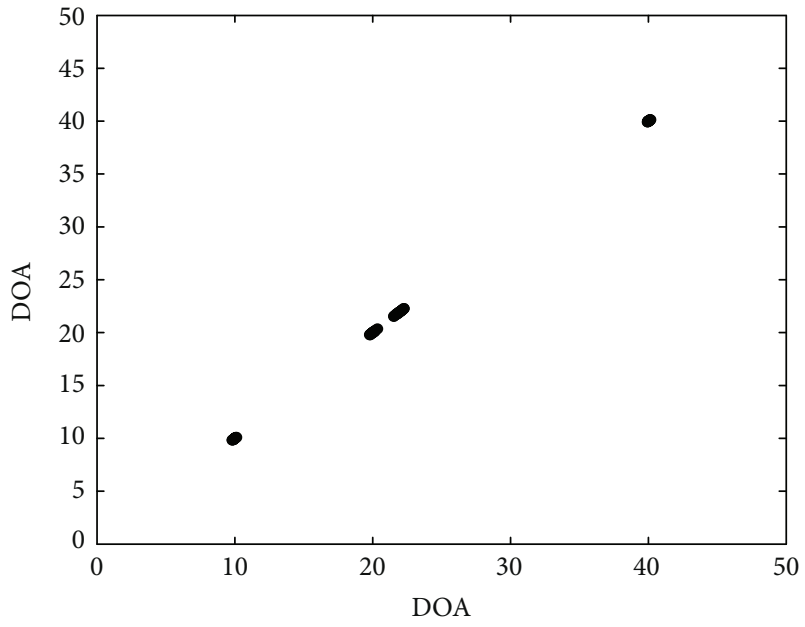

Figure 3: Angle estimation result of close sources for 30 independent trials, $\mathrm{SNR}=15 \mathrm{~dB}$.

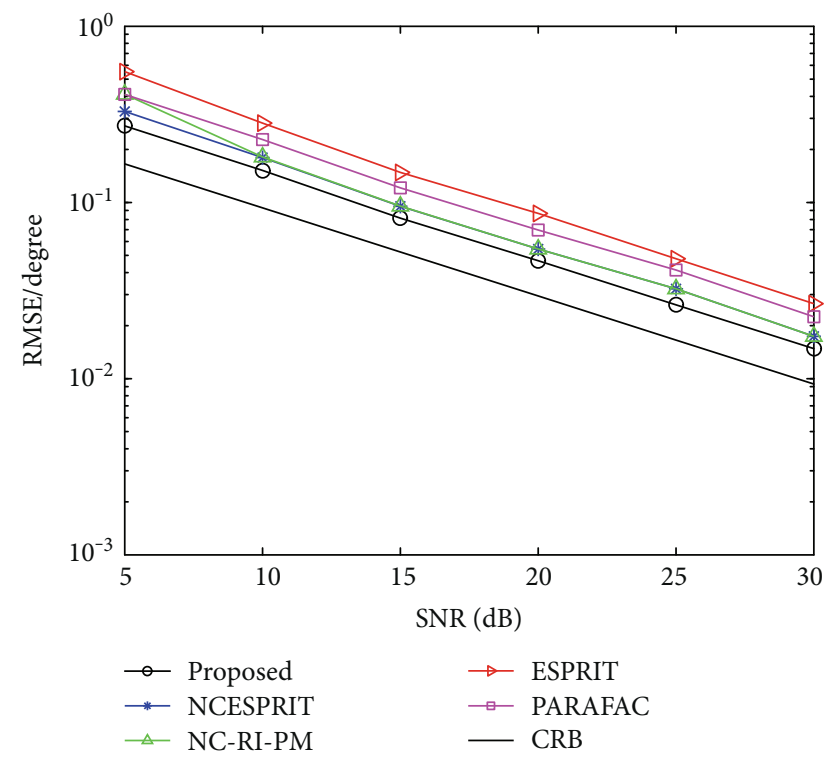

FIGURE 4: RMSE performance of different methods versus SNR.

and TALS-based PARAFAC method. In this simulation, SNR is fixed at $15 \mathrm{~dB}, M=8, K=3,\left(\theta_{1}, \theta_{2}, \theta_{3}\right)=\left(10^{\circ}, 20^{\circ}\right.$, $\left.40^{\circ}\right),\left(\psi_{1}, \psi_{2}, \psi_{3}\right)=\left(20^{\circ}, 30^{\circ}, 60^{\circ}\right)$, while $N$ is varied from 50 to 300 . We can see that average run time of each method grows as the number of snapshots $N$ increases. It can be also observed that the Vandermonde constrained PARAFAC method is more computationally efficient than the TALSbased PARAFAC method.

At last, the impact of the number of sources $K$ is investigated. Figure 7 presents the RMSE performance of our Vandermonde constrained PARAFAC method versus SNR with different $K$, when $M=8$ and $N=300$. It is observed from Figure 7 that the estimation accuracy decreases as the source number $K$ increases.

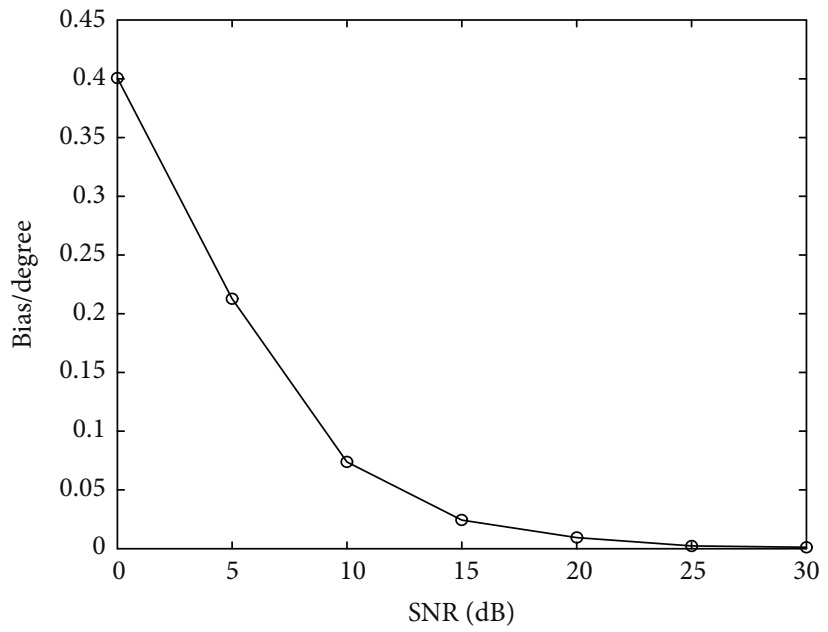

FIgURe 5: Bias of the estimator versus SNR.

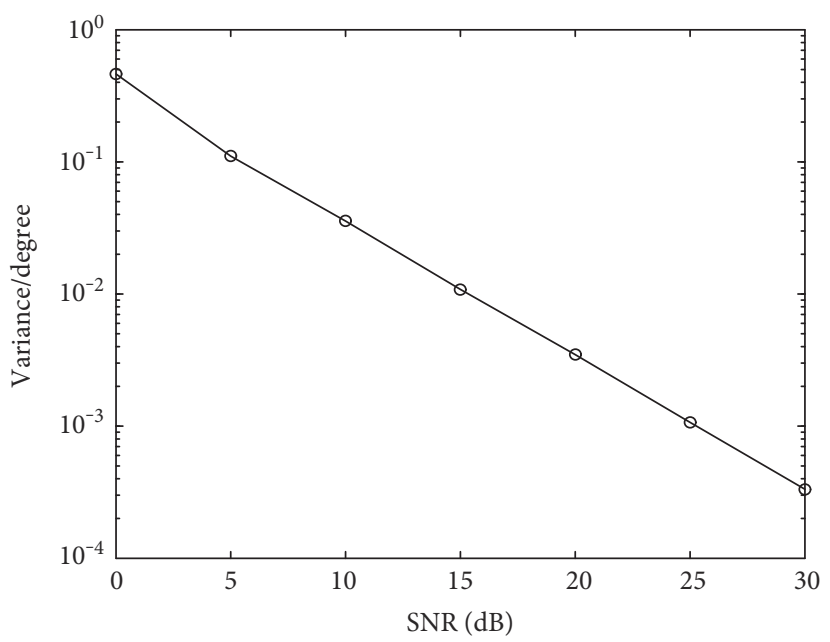

FIgURE 6: Variance of the estimator versus SNR.

\section{Conclusions}

The problem of DOA estimation of noncircular signals for uniform linear array was considered in this paper. Due to the property of noncircular signals, we constructed an extended matrix by concatenating the received data and its conjugated component. And then, a Vandermonde constrained PARAFAC model was derived from the extended matrix. Finally, estimation of the angle information was obtained via eigenvalue decomposition of two submatrices. Compared with some existing methods, the proposed method consistently provided a better DOA estimation performance. Simulation result illustrates the effectiveness of our method in terms of estimation accuracy and computational complexity. Utilizing the weighting subspace fitting to optimize the proposed method will be carried out in our future work. The case of mixed noncircular and circular signals will be also a topic for future research. 
TABLE 2: Average run time (s) of respective algorithms.

\begin{tabular}{lcccccc}
\hline$N$ & 50 & 100 & 150 & 200 & 250 & 300 \\
\hline TALS-based PARAFAC & 0.0641 & 0.0945 & 0.1120 & 0.1265 & 0.1397 & 0.1915 \\
Vandermonde constrained PARAFAC & 0.0068 & 0.0080 & 0.0093 & 0.0112 & 0.0143 & 0.0176 \\
\hline
\end{tabular}

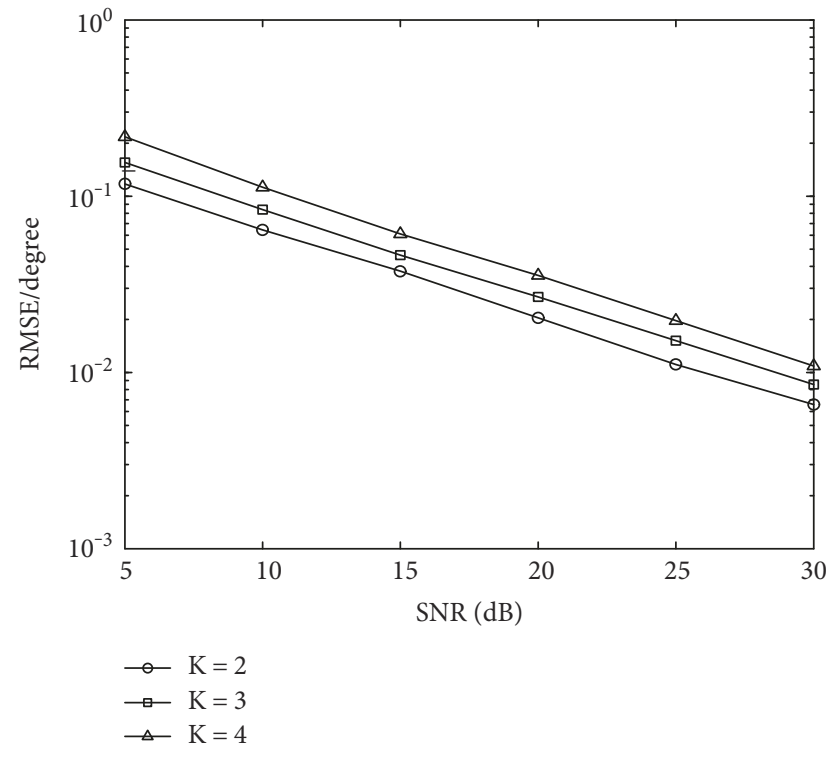

FIGURE 7: RMSE performance of the proposed method for different number of sources $K(M=8, N=300)$.

\section{Conflicts of Interest}

The authors declare that there is no conflict of interests regarding the publication of this paper.

\section{Acknowledgments}

This work was supported by the National High Technology Research and Development Program of China (863 Program, nos. 2015AA01A705 and 2014AA01A701), the National Natural Science Foundation of China (no. 61601414), and the Science and Technology Project of State Grid Corporation of China (no. SGLNDK00KJJS1700200).

\section{References}

[1] A. B. Gershman, M. Rbsamen, and M. Pesavento, "One- and two-dimensional direction-of-arrival estimation: an overview of search-free techniques," Signal Processing, vol. 90, no. 5, pp. 1338-1349, 2010.

[2] B. R. Jackson, S. Rajan, B. J. Liao, and S. Wang, "Direction of arrival estimation using directive antennas in uniform circular arrays," IEEE Transactions on Antennas and Propagation, vol. 63, no. 2, pp. 736-747, 2015.

[3] F. Gao and A. B. Gershman, "A generalized ESPRIT approach to direction-of-arrival estimation," IEEE Signal Processing Letters, vol. 12, no. 3, pp. 254-257, 2005.
[4] S. Marcos, A. Marsal, and M. Benidir, "The propagator method for source bearing estimation," Signal Processing, vol. 42, no. 2, pp. 121-138, 1995.

[5] W. H. Fang, Y. C. Lee, and Y. T. Chen, "Maximum likelihood 2-D DOA estimation via signal separation and importance sampling," IEEE Antennas and Wireless Propagation Letters, vol. 15, pp. 746-749, 2016.

[6] M. P. Clark and L. L. Scharf, "Two-dimensional modal analysis based on maximum likelihood," IEEE Transactions on Signal Processing, vol. 42, no. 6, pp. 1443-1452, 1994.

[7] N. D. Sidiropoulos, R. Bro, and G. B. Giannakis, "Parallel factor analysis in sensor array processing," IEEE Transactions on Signal Processing, vol. 48, no. 8, pp. 2377-2388, 2000.

[8] R. Roy and T. Kailath, "ESPRIT-estimation of signal parameters via rotational invariance techniques," IEEE Transactions on Acoustics, Speech, and Signal Processing, vol. 37, no. 7, pp. 984-995, 1989.

[9] R. O. Schmidt, "Multiple emitter location and signal parameter estimation," IEEE Transactions on Antennas and Propagation, vol. 34, no. 3, pp. 276-280, 1986.

[10] H. Abeida and J. P. Delmas, "MUSIC-like estimation of direction of arrival for noncircular sources," IEEE Transactions on Signal Processing, vol. 54, no. 7, pp. 2678-2690, 2006.

[11] A. Zoubir, P. Charg, and Y. Wang, "Non circular sources localization with ESPRIT," in Proceedings of the 6th European Conference on Wireless Technology (ECWT 03), Munich, Germany, October 2003.

[12] M. Haardt and F. Romer, "Enhancements of unitary ESPRIT for non-circular sources," in Proceedings of the IEEE International Conference on Acoustics, Speech, and Signal Processing (ICASSP 2004), pp. II-101-II-104, Montreal, Canada, August 2004.

[13] X. Chen, C. Wang, and X. Zhang, "DOA and noncircular phase estimation of noncircular signal via an improved noncircular rotational invariance propagator method," Mathematical Problems in Engineering, vol. 2015, Article ID 235173, 12 pages, 2015.

[14] L. Zhang, W. Lv, X. Zhang, and S. Li, "2D-DOA estimation of noncircular signals for uniform rectangular array via NCPARAFAC method," International Journal of Electronics, vol. 103, no. 11, pp. 1839-1856, 2016.

[15] R. Harshman, "Foundations of the PARAFAC procedure: models and conditions for an "explanatory" multimodal factor analysis," UCLA Working Papers in Phonetics, vol. 16, pp. 184, 1970.

[16] L. Huang, T. Long, E. Mao, and H. C. So, "MMSE-based MDL method for robust estimation of number of sources without eigendecomposition," IEEE Transactions on Signal Processing, vol. 57, no. 10, pp. 4135-4142, 2009.

[17] H. T. Wu, J. F. Yang, and F. K. Chen, "Source number estimator using Gerschgorin disks," in Proceedings of the IEEE International Conference on Acoustics, Speech and Signal Processing (ICASSP 94), pp. IV/261-IV/264, Adelaide, Australia, April, 1994. 
[18] B. Picinbono, “On circularity," IEEE Transactions on Signal Processing, vol. 42, no. 12, pp. 3473-3482, 1994.

[19] J. Du, C. Yuan, P. Tian, and H. Lin, "Channel estimation for multi-input multi-output relay systems using the PARATUCK2 tensor model," IET Communications, vol. 10, no. 9, pp. 995-1002, 2016.

[20] D. Nion and L. De Lathauwer, "A block component modelbased blind DS-CDMA receiver," IEEE Transactions on Signal Processing, vol. 56, no. 11, pp. 5567-5579, 2008.

[21] N. D. Sidiropoulos and X. Liu, "Identifiability results for blind beamforming in incoherent multipath with small delay spread," IEEE Transactions on Signal Processing, vol. 49, no. 1, pp. 228-236, 2001.

[22] G. Favier and A. de Almeida, "Overview of constrained PARAFAC models," EURASIP Journal on Advances in Signal Processing, vol. 2014, p. 40, 2014.

[23] M. Sørensen and L. De Lathauwer, "Blind signal separation via tensor decomposition with Vandermonde factor: canonical polyadic decomposition," IEEE Transactions on Signal Processing, vol. 61, no. 22, pp. 5507-5519, 2013.

[24] M. Sørensen and L. De Lathauwer, "Tensor decompositions with Vandermonde factor and applications in signal processing," in Proceedings of conference record of the forty sixth Asilomar conference on signals, systems and computers (Asilomar), pp. 890-894, Pacific Grove, CA, USA, November 2012.

[25] F. Gao, A. Nallanathan, and Y. Wang, "Improved MUSIC under the coexistence of both circular and noncircular sources," IEEE Transactions on Signal Processing, vol. 56, no. 7, pp. 3033-3038, 2008.

[26] J. Steinwandt, F. Roemer, and M. Haardt, "ESPRIT-type algorithms for a received mixture of circular and strictly non-circular signals," in Proceedings of the IEEE International Conference on Acoustics, Speech, and Signal Processing (ICASSP 2015), pp. 2809-2813, Brisbane, Australia, April 2015.

[27] J. B. Kruskal, “Three-way arrays: rank and uniqueness of trilinear decompositions, with application to arithmetic complexity and statistics," Linear Algebra and its Applications, vol. 18, no. 2, pp. 95-138, 1977.

[28] N. D. Sidiropoulos and R. Bro, "On the uniqueness of multilinear decomposition of N-way arrays," Journal of Chemometrics, vol. 14, no. 3, pp. 229-239, 2000.

[29] S. Vanhuffel, H. Chen, C. Decanniere, and P. Vanhecke, "Algorithm for time-domain NMR data fitting based on total least squares," Journal of Magnetic Resonance, Series A, vol. 110, no. 2, pp. 228-237, 1994.

[30] J. M. Papy, L. De Lathauwer, and S. Van Huffel, "Exponential data fitting using multilinear algebra: the single-channel and multi-channel case," Numerical Linear Algebra with Applications, vol. 12, no. 8, pp. 809-826, 2005.

[31] G. H. Golub and C. F. van Loan, Matrix Computations, Johns Hopkins University Press, Baltimore, 1996.

[32] Y. Rong, S. A. Vorobyov, A. B. Gershman, and N. D. Sidiropoulos, "Blind spatial signature estimation via timevarying user power loading and parallel factor analysis," IEEE Transactions on Signal Processing, vol. 53, no. 5, pp. 16971710, 2005.

[33] P. Stoica and R. L. Moses, Spectral Analysis of Signals, Pearson Prentice Hall, Upper Saddle River, NJ, USA, 2005.
[34] M. Viberg and B. Ottersten, "Sensor array processing based on subspace fitting," IEEE Transactions on Signal Processing, vol. 39, no. 5, pp. 1110-1121, 1991.

[35] A. L. Swindlehurst and T. Kailath, "Azimuth/elevation direction finding using regular array geometries," IEEE Transactions on Aerospace and Electronic Systems, vol. 29, no. 1, pp. 145-156, 1993. 


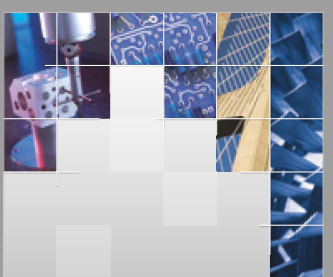

\section{Enfincering}
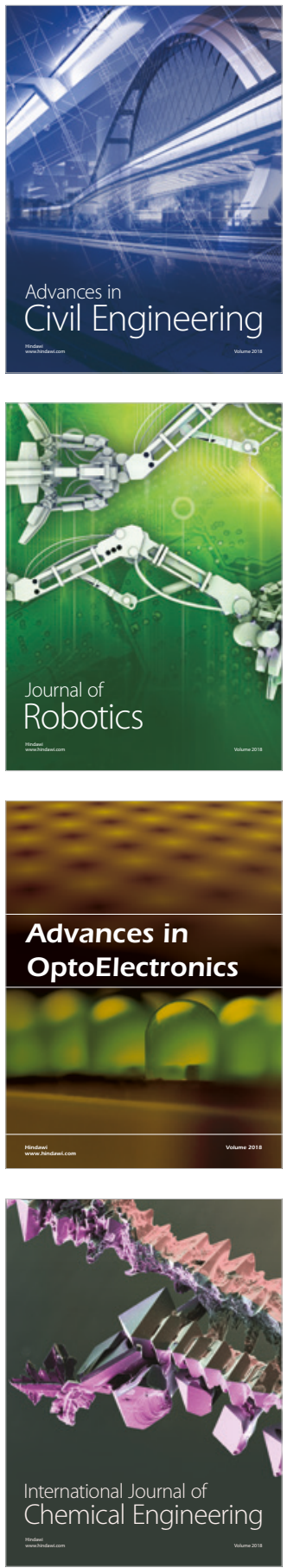

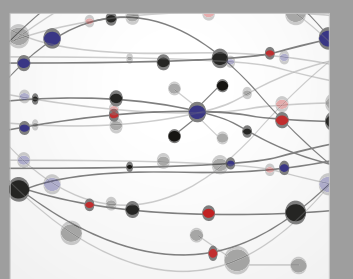

\section{Rotating \\ Machinery}

The Scientific World Journal

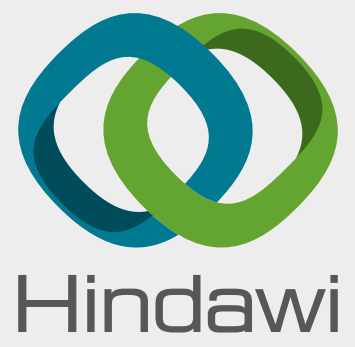

Submit your manuscripts at

www.hindawi.com
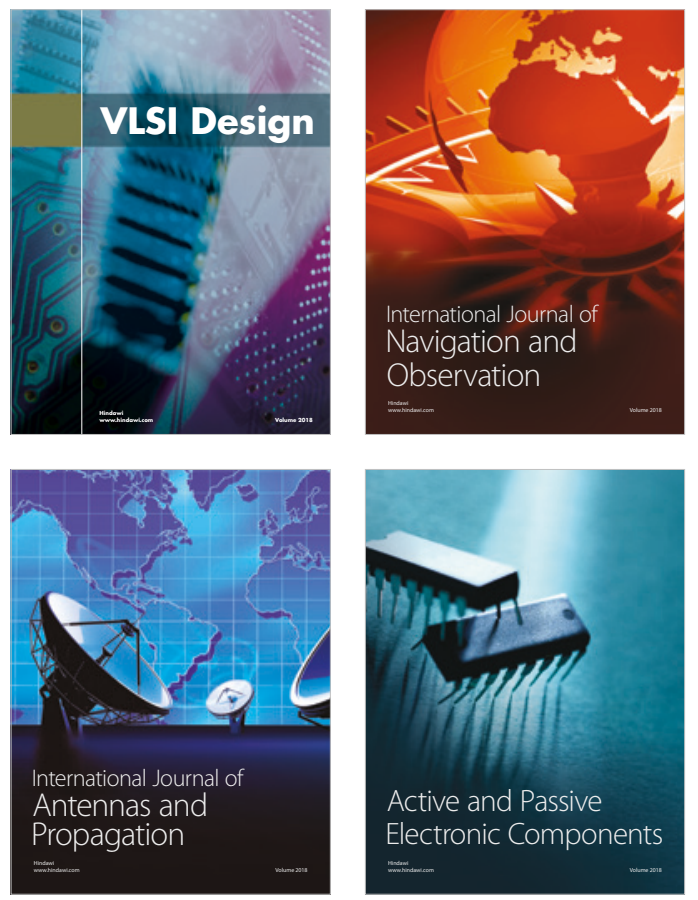
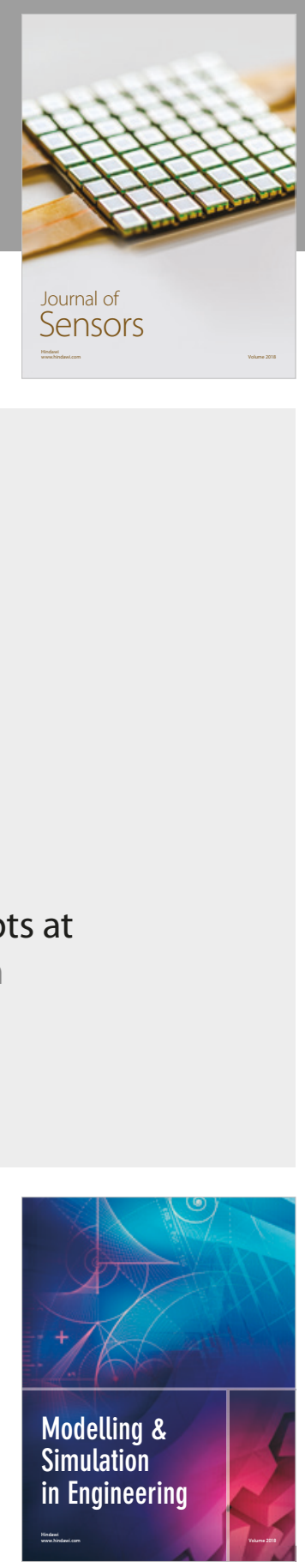

\section{Advances \\ Multimedia}
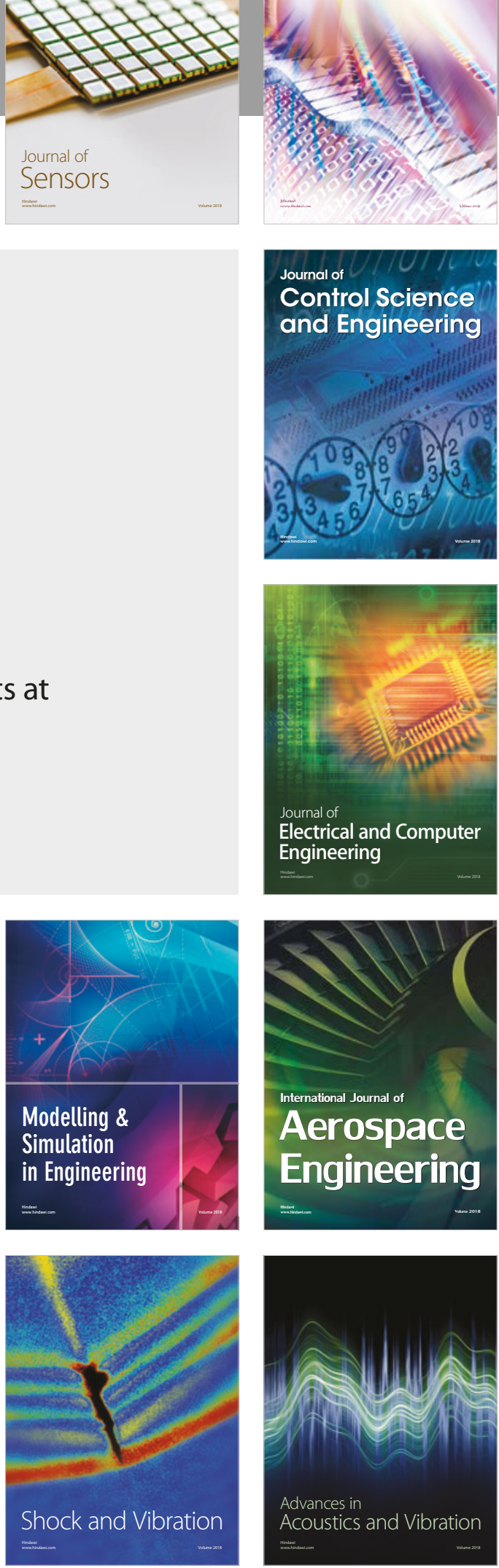